\title{
JOINT ESTIMATION OF STATES AND PARAMETERS OF LINEAR SYSTEMS WITH PARAMETER FAULTS UNDER NON-GAUSSIAN NOISES
}

$$
\text { UDC ((621.391:515.226)+681.5) }
$$

\section{Vladimir Stojanović, Dragan Pršić, Ljubiša Dubonjić}

University of Kragujevac, Faculty of Mechanical and Civil Engineering in Kraljevo, Department of Automatic Control, Robotics and Fluid Technique, Republic of Serbia

\begin{abstract}
Joint estimation of states and time-varying parameters of linear state space models is of practical importance for the fault diagnosis and fault tolerant control. Previous works on this topic consider the joint estimation in the Gaussian noise environment, but not in the presence of outliers. The known fact is that the measurements have inconsistent observations with the largest part of the observation population (outliers). They can significantly make worse the properties of linearly recursive algorithms which are designed to work in the presence of Gaussian noises. This paper proposes the strategy of the joint parameter-state robust estimation of linear state space models in the presence of non-Gaussian noises. The case of parameterdependent matrices is considered. Because of its good features in robust filtering, the extended Masreliez-Martin filter represents a cornerstone for realization of the robust algorithms for joint state-parameter estimation of linear time-varying stochastic systems in the presence of non-Gaussian noises. The good features of the proposed robust algorithm for joint estimation of linear time-varying stochastic systems are illustrated by intensive simulations.
\end{abstract}

Key words: linear stochastic systems, time-varying parameters, joint estimation, robust identification, non-Gaussian noises

\section{INTRODUCTION}

It is well known that it is very difficult to determine a large number of physical parameters which are integral part of complex systems. Despite the fact that many system parameters are available with some reasonable accuracy, a large number of parameters are known within a certain range, while some parameters are entirely unknown because

Received June 28, 2019

Corresponding author: Vladimir Stojanović

Faculty of Mechanical and Civil Engineering in Kraljevo, Department of Automatic Control, Robotics and Fluid Technique, Dositejeva 19, 36000 Kraljevo, Republic of Serbia

E-mail: stojanovic.v@mfkv.kg.ac.rs 
manufacturers consider these data as proprietary information. For example, it is difficult to ensure precise determination of system parameters such as dimensions of certain components, leakage coefficients, friction coefficients, as well as static and dynamic friction forces due to the impossibility of their direct measurement or calculation and this causes great difficulty in control of pneumatic actuators [[1]]. More precise knowledge of the system parameters increases the model quality, which causes better control performances. Hence, states filtering as well as parameters estimation can be key factors for performances, stability and accuracy of the systems.

Since Rudolf Kalman published his famous paper [[2]], the Kalman filter (KF) has become the basis of many estimation processes in different application areas. In recent years, KF has encountered a renewed interest, due to an increasing range of applications [[3],[4]]. Precise knowledge of system parameters and states is crucial for successful realization of many control techniques. Many modern engineering applications such as autonomous vehicles [[5]], strain prediction for fatigue [[6]] or robotic manipulation tasks [[7]] require real-time Kalman filtering framework with linear models.

It is usually too expensive to measure directly the system states. Self-applied state estimation methods assume that the system parameters are constant. In the real world, these parameters are always variable e.g. friction coefficients, temperature, pressure, or flow). The states estimation procedure with constant parameters will result in large errors when changing parameters. It is also known that the dynamic behaviour of complex systems is usually described by a linear stochastic state space model with time-varying parameters [[8], [9]]. Therefore, methods by which parameter and state estimation can be obtained at the same time are required.

A significant number of papers have been published on the theme of the Kalman filter application for parameters estimation of dynamic systems. One of the first papers dealing with this topic is [[10]]. The convergence analysis of the extended Kalman filter for parameters estimation was analyzed in [[11]]. The joint estimation of states and parameters using the Kalman filters is widespread [[12], [13], [14]]. The joint estimation of states and time-varying parameters is of great practical importance for fault diagnosis and fault tolerant control [[15]]. One of the biggest challenges in the design of flight control systems is a requirement for the flight of the aircraft to recover safely from structural damage and/or system faults. Regardless of whether the aircraft is equipped with a special control reconfiguration capability, reliable fault diagnostic information is extremely important to the pilot. The main challenge is the detection and isolation of incipient faults in the presence of modeling uncertainty and noise [[16]]. The problem of the joint estimation in dynamic systems has been intensively studied and the basic estimation techniques are well established, but, because of the complex nature of dynamic systems, the application of techniques to these problems is not straightforward. These methods are quite popular. Inclusion of unknown parameters in the state vector allows easy implementation of the estimation algorithm, because the problem of parameters estimation in this case is solved using the standard filtering theory. In this way, a good joint estimation technique was obtained. Also, thus derived recursive estimation algorithm enables both offline and online realization.

On the other side, there is no such solution available for linear systems in the presence of non-Gaussian measurements. Because of that, in this paper, it is assumed that the measurement noise is non-Gaussian. Justification of this approach was confirmed in practice [[17]]. Namely, the known fact is that the measurements have inconsistent observations with 
the largest part of the observation population (outliers). Their presence can destroy the good features of linearly recursive algorithms which are designed for estimation in the presence of Gaussian noises. Therefore, it is very important to design a robust algorithm which would be a little sensitive to outliers. The Huber's theory of robust statistics is crucial for the algorithm design whose robustness is achieved by introducing a nonlinear transformation of prediction error (Huber's function) [[18], [19]].

The Masreliez-Martin filter is a frame for realization of the described algorithms. It is considered the case when the process noise has a Gaussian distribution, and the measurement noise has a non-Gaussian distribution. Some heuristic modifications have been made in order to improve features of the robust filters. Namely, in a posteriori filter matrix, Fisher information has been replaced with a derivative of the Huber's function. The benefits of such modified filters have been shown in [[20]]. Because of its good properties in robust filtering, the modified extended Masreliez-Martin filter represents a cornerstone for realization of the robust algorithm for the joint state-parameter estimation of linear time-varying stochastic systems in the presence of non-Gaussian noises.

Considering the unknown parameters of the dynamic system, for the purpose of pursuing the accuracy robust estimation, this paper proposes two kinds of strategies to estimate the state and parameter jointly. The state estimation and parameter identification are united by the joint parameter and state estimation. It is completely natural to put unknown, generally time-varying, parameters in the vector of states, after which the problem is reduced to the classic filtering problem. This paper considers linear state space systems with matrices which are parameter dependent. In that case, in order to obtain the robust algorithm for the joint estimation of states and parameters, we shall consequently face with a general nonlinear filtering problem. Conventionally, the joint state-parameter robust estimation problem in such systems is based on the extended Kalman filter.

The good features of the proposed robust algorithms are illustrated through intensive simulations.

\section{Joint Estimation Algorithm in CASE OF PARAMETER DEPENDENT MATRICES}

In this paper, we consider state-space systems with time-varying parameters in the following form:

$$
\begin{gathered}
x(k+1)=A(\theta(k)) x(k)+B(\theta(k)) u(k)+w(k) \\
y(k)=D(\theta(k)) x(k)+e(k)
\end{gathered}
$$

where $x(k) \in R^{n}$ and $\theta(k) \in R^{p}$ are unknown state and parameter vectors, respectively. From a practical point of view, it is unreasonable to make assumptions about the fault characteristics and not to consider these as unknown time functions. The general form of parameters changing of the stochastic linear system is defined as follows:

$$
\theta(k+1)=G \theta(k)+\eta(k)
$$

in which $G$ is a priori known nonsingular matrix which is convenient for inclusion of a priori information on the phenomenon which is identified. The stochastic process $\eta(k)$ is zero-mean white noise whose covariance matrix $\Phi(k)$ has the form: 


$$
\eta(k): N(0, \Phi(k))
$$

Input and measured output vector of the system are $u(k) \in R^{m}$ and $y(k) \in R^{r}$. It is assumed that the process noise is the zero-mean Gaussian white noise $w(k): \mathcal{N}(0, Q(k))$, in which $Q(k)$ is the covariance matrix. The measurement noise $e(k)$ has a nonGaussian distribution with approximately normal distribution classes:

$$
\mathscr{P}_{\varepsilon}=\left\{p(e): p(e)=(1-\varepsilon) p_{1}(e)+\varepsilon p_{2}(e)\right\}
$$

in which the probability density $p(e)$ represents a mixture of the primary probability density $p_{1}(e): \mathcal{N}\left(0, R_{1}(k)\right)$ and the contaminating probability density $p_{2}(e): \mathcal{N}\left(0, R_{2}(k)\right)$ where the contamination degree $\varepsilon$ is in the range $0<\varepsilon<1$, while $R_{1}(k)$ and $R_{2}(k)$ are covariance matrices of primary and contaminating terms in the non-Gaussian distribution (5), respectively.

In some cases, the fault $f_{p}(\mathrm{k})$ could be expressed as a change in the system parameter, for example a change in the $i_{t h}$ row and $j_{t h}$ column element of the matrix $A$, the system can then be described as (1)-(2), see Fig. 1.

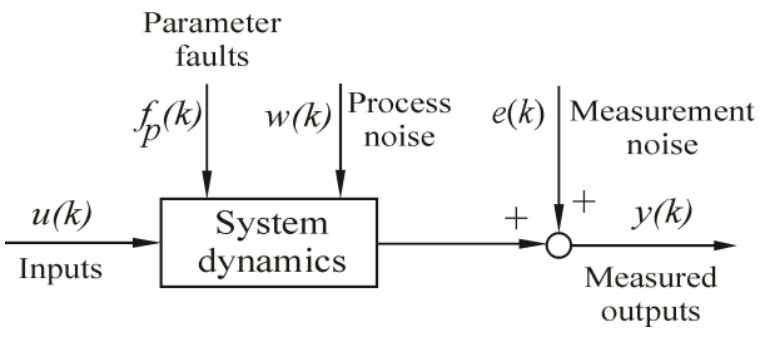

Fig. 1 Open-loop system with parameter faults in the system

This approach is based on the assumption that the faults are reflected in the physical system parameters such as friction, mass, viscosity, resistance, inductance, capacitance, etc. As indicated, the linear state space model is often specified up to the value of some parameters $\theta(k)$. Since matrices $A, B$ and $D$ are dependent of parameters $\theta(k)$ and due to multiplying with state vector $x(k)$, the system (1)-(2) is nonlinear. Hence, to obtain the parameter estimation recursively, we shall consequently face a general nonlinear filtering problem.

Our goal is to derive the robust algorithm for the joint state and parameter estimation of stochastic linear systems in the presence of outliers which maintains a low sensitivity in appearance of outliers. Using the joint state-parameter formulation, a unified estimation of states and parameters has been achieved. It is completely natural to put the parameters in the vector of states, after which the problem is reduced to the classic filtering problem of $k$-th order unified system (where $k=n+p$, in which $n$ is a number of estimated states and $p$ is a number of estimated parameters).

The robust extended Kalman filter can be used to estimate states and parameters of linear stochastic systems, after the joint state-parameter formulation: 


$$
z(k)=\left[\begin{array}{c}
x(k) \\
\theta(k)
\end{array}\right]=\left[\begin{array}{c}
f_{k-1}(x(k-1), u(k-1), \theta(k-1)) \\
g_{k-1}(\theta(k-1))
\end{array}\right]+\left[\begin{array}{c}
w(k-1) \\
\eta(k-1)
\end{array}\right]
$$

The extended system is given in a more compact form:

$$
\begin{gathered}
z(k)=q_{k-1}(z(k-1), u(k-1), \theta(k-1))+\xi(k-1) \\
y(k)=h_{k}(z(k), \theta(k))+e(k)
\end{gathered}
$$

where $z(k)$ denotes the extended state vector $z(k)=\left[\begin{array}{lll}x^{T}(k) & \theta^{T}(k)\end{array}\right]^{T}, \xi(k-1)$ represents the extended disturbance vector $\xi(k-1)=\left[\begin{array}{ll}w^{T}(k-1) & \eta^{T}(k-1)\end{array}\right]^{T}$, in which

$$
\xi(k): N(0, \Xi(k)) \text { and } \Xi(k)=\left[\begin{array}{cc}
Q(k) & 0 \\
0 & \Phi(k)
\end{array}\right] .
$$

Based on the extended robust filter which is proposed in [[20]], the robust algorithm for joint states-parameters estimation of system (7)-(8) has the following form:

$$
\begin{gathered}
\hat{z}(k \mid k-1)=q_{k-1}(\hat{z}(k-1 \mid k-1), u(k-1), \theta(k-1), 0) \\
P(k \mid k-1)=F(k-1) P(k-1 \mid k-1) F^{T}(k-1)+L(k-1) \Xi(k-1) L^{T}(k-1) \\
K(k)=P^{T}(k \mid k-1) H^{T}(k) T^{T}(k) \\
v(k)=T(k)\left[y(k)-h_{k}(\hat{z}(k \mid k-1), \theta(k), 0)\right] \\
\hat{z}(k \mid k)=\hat{z}(k \mid k-1)+K(k) \Psi(v(k)) \\
P(k \mid k)=P(k \mid k-1)-K(k) \Psi^{\prime}(v(k)) K^{T}(k) \\
\Psi^{\prime}(v(k))=\left[\begin{array}{ccc}
\psi^{\prime}\left(v_{1}(k)\right) & \ldots & 0 \\
0 & \ddots & 0 \\
0 & \ldots & \psi^{\prime}\left(v_{r}(k)\right)
\end{array}\right] \\
T(k)=\left[H(k) P(k \mid k-1) H^{T}(k)+V(k) R_{1}(k) V^{T}(k)\right]^{-\frac{1}{2}}
\end{gathered}
$$

where

$$
\begin{aligned}
& F(k)=\left.\frac{\partial q_{k}}{\partial z}\right|_{\hat{z}(k \mid k)}=\left[\begin{array}{c:c}
\frac{\partial f_{k}}{\partial x} & \frac{\partial f_{k}}{\partial \theta} \\
\hdashline 0 & I_{p}
\end{array}\right]_{\hat{x}(k \mid k), \hat{\theta}(k \mid k)}=\left[\begin{array}{c:c}
A(k) & F_{\theta}(k) \\
\hdashline 0 & I_{p}
\end{array}\right], \quad L(k)=\left.\frac{\partial q_{k}}{\partial \xi}\right|_{\hat{z}(k \mid k)}=I_{n+p} \\
& H(k)=\left.\frac{\partial h_{k}}{\partial z}\right|_{\hat{z}(k \mid k-1)}=\left[\begin{array}{l:l:l}
\frac{\partial h_{k}}{\partial x} & \frac{\partial h_{k}}{\partial \theta}
\end{array}\right]_{\hat{x}(k \mid k-1), \hat{\theta}(k \mid k-1)}=\left[D(k): H_{\theta}(k)\right], V(k)=\left.\frac{\partial h_{k}}{\partial v}\right|_{\hat{z}(k \mid k-1)}=I_{r}
\end{aligned}
$$


Initial conditions are:

$$
\hat{z}_{0}=0 \text { and } P_{0}=\left[\begin{array}{c:c}
P\left(x_{0}\right) & 0 \\
\hdashline 0 & P\left(\theta_{0}\right)
\end{array}\right]
$$

Here $\hat{z}(k)$ is the estimate of extended state vector, and generally, $P(k)$ denotes the filter covariance matrix $P(k)=E\left\{(\hat{x}(k)-x(k))(\hat{x}(k)-x(k))^{T}\right\}$. In relation (13), $v(k)$ represents transformed residuals $\varepsilon(k)=y(k)-H(k) \hat{z}(k-1 \mid k-1)$. In order to fulfill the conditions of symmetry of certain probability densities and conditions for marginal probabilities, the transformation $T(k)$ has been included in the residual [[19]].

For the class of $\varepsilon$-contaminated distributions of probabilities, the nonlinear transformation of prediction error $\psi(\cdot)$ (Huber's function), is obtained using the game theory in statistics [[18]]

$$
\psi(v(k))=\min \left\{\left|v(k), k_{\varepsilon}\right|\right\} \operatorname{sgn}(v(k))
$$

and its derivative:

$$
\psi^{\prime}(v(k))= \begin{cases}1 & |v(k)|<k_{\varepsilon} \\ 0 & \text { otherwise. }\end{cases}
$$

in which $k_{\varepsilon}$ is appropriately defined parameter of the Huber's function, see Fig. 2.

The following relation determines the relationship between the contamination degree $\varepsilon$ and the parameter $k_{\varepsilon}$ of the Huber's function [[18]]:

$$
\frac{\Phi_{N}^{\prime}\left(k_{\varepsilon}\right)}{k_{\varepsilon}}-2 \Phi_{N}\left(-k_{\varepsilon}\right)=\frac{\varepsilon}{1-\varepsilon}, \Phi_{N}(x)=\frac{1}{\sqrt{2 \pi}} \int_{-\infty}^{x} e^{-\frac{y^{2}}{2}} d y
$$

in which $\Phi_{N}$ represents the standard normal cumulative distribution function.
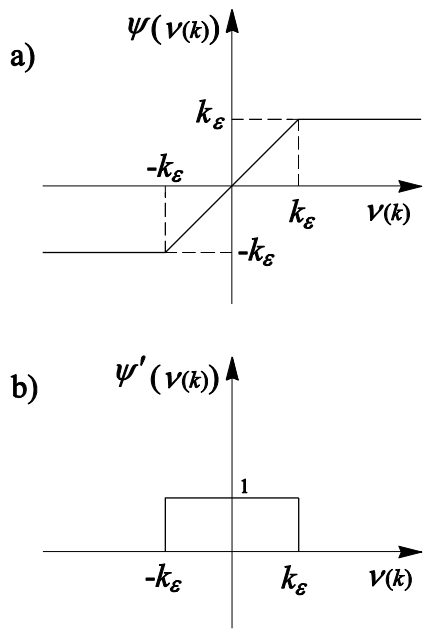

Fig. 2 Huber's function and its derivative 
It can be shown that the mathematical expectation and the covariance matrix of transformed residuals $\varepsilon(\mathrm{k})=y(k)-H(k) \hat{x}(k-1 \mid k-1)$ are:

$$
\begin{gathered}
E\{v(k)\}=T(k) E\{\varepsilon(k)\}=0 \\
E\left\{v^{2}(k)\right\}=T^{2}(k)\left[H(k) P(\mathrm{k} \mid k-1) H^{T}(k)+R_{1}(k)\right]^{-\frac{1}{2}}
\end{gathered}
$$

Therefore, in the case that transformation $T(k)$ is:

$$
T(k)=\left[H(k) P(\mathrm{k} \mid k-1) H^{T}(k)+R_{1}(k)\right]^{-1 / 2}
$$

the covariance matrix of the transformed residuals will be $I$.

The following block structure of the matrix gain and covariance matrices is in accordance with the joint state-parameter formulation (6):

$$
\begin{gathered}
K(k)=\left[\begin{array}{c}
N(k) \\
\hdashline M(k)
\end{array}\right], P(k \mid k-1)=\left[\begin{array}{c:c}
P_{1}(k \mid k-1) & P_{2}(k \mid k-1) \\
\hdashline P_{2}^{T}(k \mid k-1) & P_{3}(k \mid k-1)
\end{array}\right] \\
P(k \mid k)=\left[\begin{array}{c:c}
P_{1}(k \mid k) & P_{2}(k \mid k) \\
\hdashline P_{2}^{T}(k \mid k) & P_{3}(k \mid k)
\end{array}\right]
\end{gathered}
$$

Keeping this in mind, estimates of a priori and a posteriori extended state vectors, relations (10) and (14), are given by the following relations:

$$
\begin{gathered}
\hat{z}(k \mid k-1)=\left[\begin{array}{c}
f_{k-1}(\hat{x}(k-1 \mid k-1), u(k-1), \hat{\theta}(k-1 \mid k-1)) \\
\hat{\theta}(k-1 \mid k-1)
\end{array}\right] \\
\hat{z}(k \mid k)=\left[\begin{array}{c}
\hat{x}(k \mid k-1) \\
\hat{\theta}(k \mid k-1)
\end{array}\right]+\left[\begin{array}{l}
N(k) \\
M(k)
\end{array}\right][\Psi(v(k))]
\end{gathered}
$$

Now, it is easy to express the estimates of the state and parameter vectors:

$$
\begin{gathered}
\hat{x}(k \mid k)=f_{k-1}(\hat{x}(k-1 \mid k-1), u(k-1), \hat{\theta}(k-1 \mid k-1))+N(k) \Psi(v(k)) \\
\hat{\theta}(k \mid k)=\hat{\theta}(k-1 \mid k-1)+M(k) \Psi(v(k))
\end{gathered}
$$

In this way, the robust algorithm for the joint states-parameters estimation of linear stochastic systems in case of parameter-dependent matrices, has been derived. The robust algorithm is summarized in Table 1. 
Table 1 Joint estimation algorithm in case of parameter-dependent matrices

Estimates of a priori vectors of states and parameters

$$
\begin{aligned}
& \hat{x}(k \mid k-1)=f_{k-1}(\hat{x}(k-1 \mid k-1), u(k-1), \hat{\theta}(k-1 \mid k-1)) \\
& \hat{\theta}(k \mid k-1)=\hat{\theta}(k-1 \mid k-1)
\end{aligned}
$$

Jacobian matrices of nonlinear functions $q_{k}$ и $h_{k}$

$$
\begin{gathered}
F(k)=\left.\frac{\partial q_{k}}{\partial z}\right|_{\hat{z}(k \mid k)}=\left[\begin{array}{c:c}
\frac{\partial f_{k}}{\partial x} & \frac{\partial f_{k}}{\partial \theta} \\
\hdashline 0 & I_{p}
\end{array}\right]_{\hat{x}(k \mid k), \hat{\theta}(k \mid k)}=\left[\begin{array}{c:c}
A(k) & F_{\theta}(k) \\
\hdashline 0 & I_{p}
\end{array}\right], \quad L(k)=\left.\frac{\partial q_{k}}{\partial \xi}\right|_{\hat{z}(k \mid k)}=I_{n+p} \\
H(k)=\left.\frac{\partial h_{k}}{\partial z}\right|_{\hat{z}(k \mid k-1)}=\left[\begin{array}{c:c}
\frac{\partial h_{k}}{\partial x} & \left.\frac{\partial h_{k}}{\partial \theta}\right]_{\hat{x}(k \mid k-1), \hat{\theta}(k \mid k-1)}=[D(k)
\end{array} H_{\theta}(k)\right], V(k)=\left.\frac{\partial h_{k}}{\partial v}\right|_{\hat{z}(k \mid k-1)}=I_{r}
\end{gathered}
$$

Matrix elements of a priori covariance matrix $P(k \mid k-1)$

$$
\begin{aligned}
& P_{1}(k \mid k-1)=A(k-1) P_{1}(k-1 \mid k-1) A^{T}(k-1)+F_{\theta}(k-1) P_{2}^{T}(k-1 \mid k-1) A^{T}(k-1)+ \\
& +A(k-1) P_{2}(k-1 \mid k-1) F_{\theta}^{T}(k-1)+F_{\theta}(k-1) P_{3}(k-1 \mid k-1) F_{\theta}^{T}(k-1)+Q(k-1) \\
& P_{2}(k \mid k-1)=A(k-1) P_{2}(k-1 \mid k-1)+F_{\theta}(k-1) P_{3}(k-1 \mid k-1) \\
& P_{3}(k \mid k-1)=P_{3}(k-1 \mid k-1)+\Phi(k-1)
\end{aligned}
$$

Transformation matrix

$$
\begin{aligned}
& T(k)=\left[D(k) P_{1}(k \mid k-1) D^{T}(k)+H_{\theta}(k) P_{2}^{T}(k \mid k-1) D^{T}(k)+\right. \\
& \left.+D(k) P_{2}(k \mid k-1) H_{\theta}^{T}(k)+H_{\theta}(k) P_{3}(k \mid k-1) H_{\theta}^{T}(k)+R_{1}(k)\right]^{-\frac{1}{2}}
\end{aligned}
$$

Matrix elements of the gain matrix $K(k)$

$$
\begin{gathered}
N(k)=P_{1}(k \mid k-1) D^{T}(k) T^{T}(k)+P_{2}(k \mid k-1) H_{\theta}^{T}(k) T^{T}(k), \\
M(k)=P_{2}^{T}(k \mid k-1) H_{x}^{T}(k) T^{T}(k)+P_{3}(k \mid k-1) H_{\theta}^{T}(k) T^{T}(k)
\end{gathered}
$$

Transformed prediction error

$$
v(k)=T(k)\left[y(k)-h_{k}(\hat{x}(k \mid k-1), \theta(k), 0)\right]
$$

Huber's matrix function

$$
\Psi(v(k))=\left[\begin{array}{llll}
\psi\left(v_{1}(k)\right) & \ldots & \psi\left(v_{r}(k)\right)
\end{array}\right]^{T}
$$

Matrix elements of a posteriori covariance matrix $P(k \mid k)$

$P_{1}(k \mid k)=P_{1}(k \mid k-1)-N(k) \Psi^{\prime}(v(k)) N^{T}(k), P_{2}(k \mid k)=P_{2}(k \mid k-1)-N(k) \Psi^{\prime}(v(k)) M^{T}(k)$,

$P_{3}(k \mid k)=P_{3}(k \mid k-1)-M(k) \Psi^{\prime}(v(k)) M^{T}(k)$ 


\begin{tabular}{l}
\hline Estimates of a posteriori vectors of states and parameters \\
$\qquad \hat{x}(k \mid k)=f_{k-1}(\hat{x}(k-1 \mid k-1), u(k-1), \hat{\theta}(k-1 \mid k-1))+N(k) \Psi(v(k))$ \\
$\qquad \hat{\theta}(k \mid k)=\hat{\theta}(k-1 \mid k-1)+M(k) \Psi(v(k))$. \\
Initial conditions \\
$\hat{x}(0 \mid 0)=0, \quad \hat{\theta}(0 \mid 0)_{0}=0, P_{1}(0 \mid 0)=P\left(x_{0}\right)=10^{4} I_{n}, P_{2}(0 \mid 0)=0, P_{3}(0 \mid 0)=P\left(\theta_{0}\right)=10^{4} I_{p}$
\end{tabular}

\section{SiMULATION RESULTS}

Since the linear state space system with parameter dependent matrices is considered, we need to apply the extended Kalman filter based algorithm. Following results demonstrate superiority of the proposed robust joint estimation algorithm in presence of non-Gaussian noises in relation to the joint estimation algorithms based on the widelyused extended Kalman filter and extended Masreliez-Martin filter. The behavior of the algorithms will be considered on:

$$
\begin{gathered}
{\left[\begin{array}{l}
x_{1}(k+1) \\
x_{2}(k+1)
\end{array}\right]=\left[\begin{array}{cc}
p_{1}(\mathrm{k}) & 0 \\
0 & p_{2}(\mathrm{k})
\end{array}\right]\left[\begin{array}{l}
x_{1}(k) \\
x_{2}(k)
\end{array}\right]+\left[\begin{array}{l}
w_{1}(k) \\
w_{2}(k)
\end{array}\right]} \\
{\left[\begin{array}{l}
y_{1}(k) \\
y_{2}(k)
\end{array}\right]=\left[\begin{array}{cc}
p_{3}(\mathrm{k}) & 0 \\
0 & 0.9
\end{array}\right]\left[\begin{array}{l}
x_{1}(k) \\
x_{2}(k)
\end{array}\right]+\left[\begin{array}{l}
e_{1}(k) \\
e_{2}(k)
\end{array}\right]}
\end{gathered}
$$

The features of the proposed robust algorithm are considered on the model whose time-varying parameter vector has an expected value:

$$
\bar{\theta}=\left[\begin{array}{lll}
1.01 & 0.98 & 0.99
\end{array}\right]^{T}
$$

The process noise $w(k)$ is the zero-mean white noise with covariance matrix:

$$
Q(\mathrm{k})=\left[\begin{array}{cc}
10^{-5} & 0 \\
0 & 10^{-5}
\end{array}\right]
$$

The covariance matrix of parameters has the form:

$$
\Phi(k)=\left[\begin{array}{ccc}
2 \cdot 10^{-6} & 0 & 0 \\
0 & 1 \cdot 10^{-6} & 0 \\
0 & 0 & 2 \cdot 10^{-6}
\end{array}\right]
$$

The non-Gaussian distribution of the measured noise is given by

$$
\mathbb{P}_{\varepsilon}=\left\{\begin{array}{l}
p\left(v_{1}\right)=\left(1-\varepsilon_{1}\right) \cdot \mathcal{N}(0 ; 0.0001)+\varepsilon_{1} \cdot \mathcal{N}(0 ; 0.01) \\
p\left(v_{2}\right)=\left(1-\varepsilon_{2}\right) \cdot \mathcal{N}(0 ; 0.0001)+\varepsilon_{2} \cdot \mathcal{N}(0 ; 0.01)
\end{array}\right\}
$$


For the purpose of illustrating the estimation quality, the mean square error (MSE) is used as follows:

$$
M S E=\ln \left(\mathrm{E}\|\hat{z}(k)-z(k)\|^{2}\right)
$$

The system outputs, estimates of states and parameters, as well as mean square errors in the case when contaminations have values $\varepsilon_{1}=\varepsilon_{2}=0.1$ are shown on Figs. 3-6.
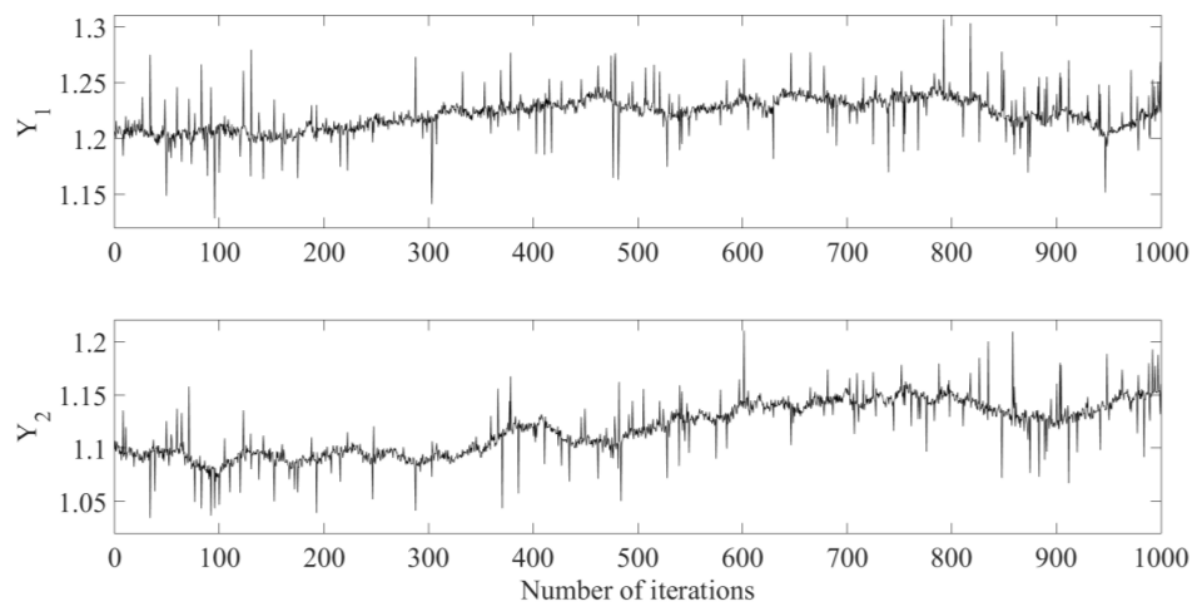

Fig. 3 System output signals
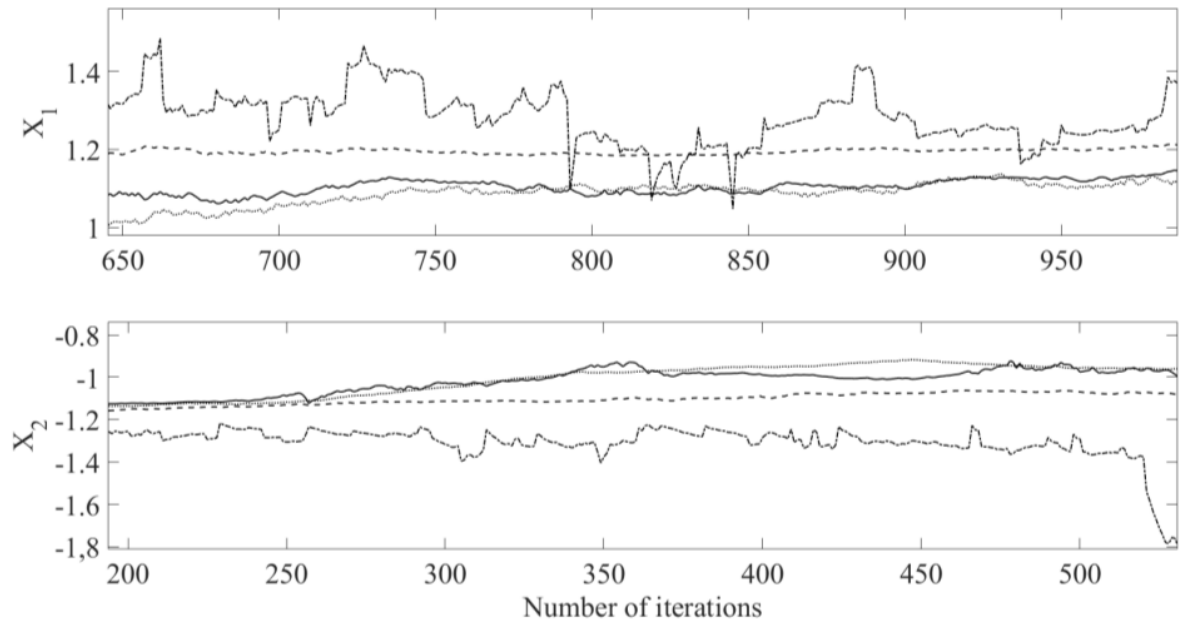

Fig. 4 States estimates (solid line: Proposed joint robust algorithm, dashed line: Joint algorithm based on extended Masreliez-Martin filter, dash-dot: Joint algorithm based on extended Kalman filter, dotted line: True values of states). 
Joint Estimation of States and Parameters of Linear Systems with Parameter Faults Under non-Gaussian Noises 123
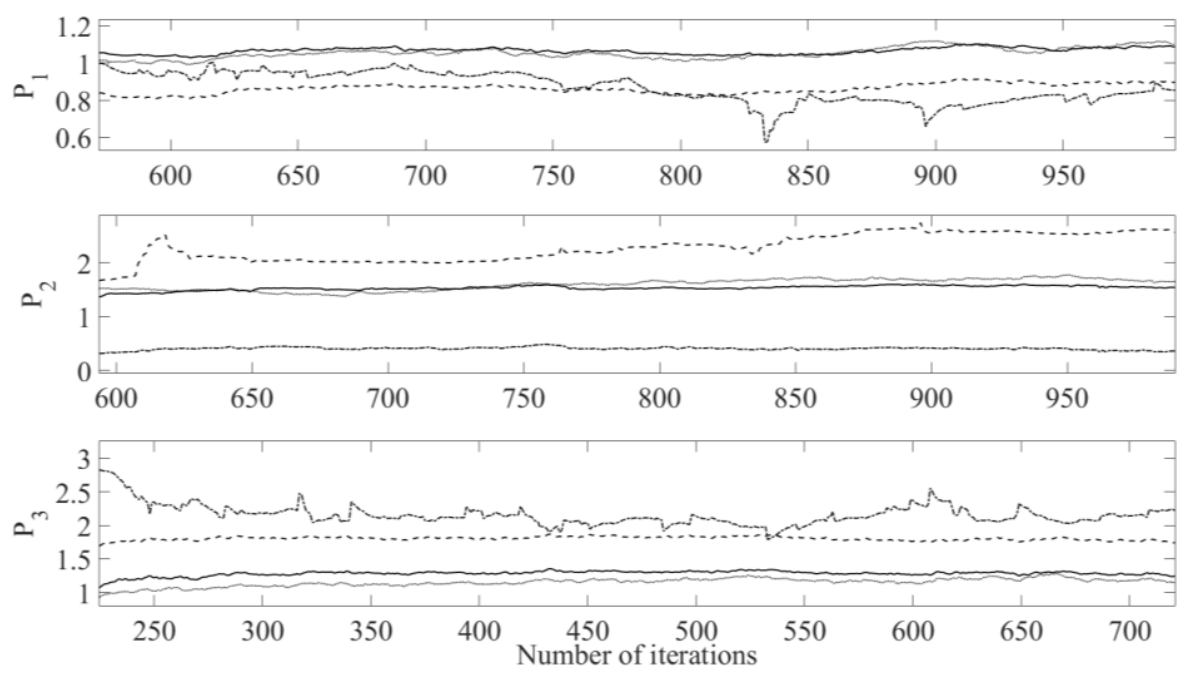

Fig. 5 Parameters estimates (solid line: Proposed robust joint algorithm, dashed line: Joint algorithm based on extended Masreliez-Martin filter, dash-dot: Joint algorithm based on extended Kalman filter, dotted line: True values of states).

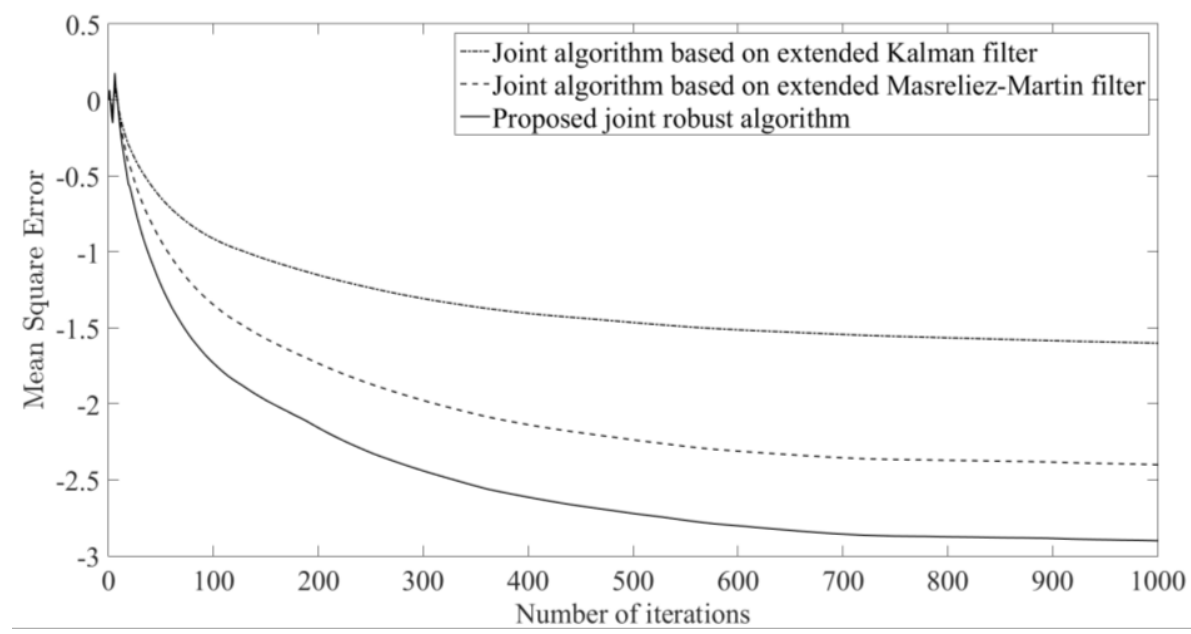

Fig. 6 Mean square errors

The presented results have shown that the widely-used extended Kalman filter (EKF) is very sensitive to the presence of non-Gaussian noises, as opposed to the proposed robust joint estimation algorithm. In order to show the robustness of the proposed robust joint estimation algorithm for systems with parameter faults in relation to other conventional and widely-used joint estimation algorithms, the algorithms are run 30 times independently, for different contamination degrees. Furthermore, it is particularly important to note that 
proposed robust estimation algorithm for systems with parameter faults (REA), see Table 2, keeps high performances in relation to joint estimation algorithms based on modern filters, such as: Gaussian sum filter (GSF), particle filter (PF) or interactive multiple modelling filter (IMMF). Table 2 provides statistical data based on 1000 iterations.

Table 2 Mean square errors for different degrees of contaminations.

\begin{tabular}{|c|c|c|c|c|}
\hline $\begin{array}{l}\text { Joint estimation algorithms for } \\
\text { systems with parameter faults }\end{array}$ & Mean & Best & Worst & Variance \\
\hline Case I & \multicolumn{4}{|c|}{ Contamination degree $\varepsilon=0.05$} \\
\hline EKF & -1.3412 & -1.5954 & -1.1495 & 0.0106 \\
\hline $\mathrm{PF}$ & -1.8512 & -1.4948 & -1.0992 & 0.0104 \\
\hline IMMF & -2.0176 & -2.2109 & -1.7591 & 0.0100 \\
\hline EMMF & -2.5143 & -2.6805 & -2.3139 & 0.0091 \\
\hline GSF & -3.1892 & -3.3863 & -2.9211 & 0.0085 \\
\hline REA & -4.1524 & -4.3158 & -3.8812 & 0.0078 \\
\hline Case II & \multicolumn{4}{|c|}{ Contamination degree $\varepsilon=0.1$} \\
\hline $\mathrm{EKF}$ & -0.9541 & -1.1633 & -0.6629 & 0.0116 \\
\hline $\mathrm{PF}$ & -1.1257 & -1.3723 & -0.8584 & 0.0112 \\
\hline IMMF & -1.6712 & -1.7898 & -1.5013 & 0.0102 \\
\hline EMMF & -2.0123 & -2.2015 & -1.7386 & 0.0094 \\
\hline GSF & -3.4125 & -3.4629 & -3.2603 & 0.0090 \\
\hline REA & -3.6542 & -3.8562 & -3.4870 & 0.0079 \\
\hline Case III & \multicolumn{4}{|c|}{ Contamination degree $\varepsilon=0.2$} \\
\hline EKF & -0.6471 & -0.8246 & -0.4043 & 0.0125 \\
\hline $\mathrm{PF}$ & -0.9932 & -1.2598 & -0.8591 & 0.0115 \\
\hline IMMF & -2.0158 & -2.2060 & -1.8200 & 0.0111 \\
\hline EMMF & -2.6521 & -2.9896 & -2.5099 & 0.0098 \\
\hline GSF & -2.9332 & -3.0394 & -2.7789 & 0.0089 \\
\hline REA & -3.3254 & -3.4645 & -3.1397 & 0.0082 \\
\hline
\end{tabular}

From Table 2, it can be seen that the worst results obtained by the proposed robust joint algorithm is even better than the best result obtained by others, at a certain contamination degree. Furthermore, from Table 2, it can be clearly seen that the superiority of the proposed robust algorithm is greater in higher degrees of contamination. Also, it is important to note that in presence of non-Gaussian noises, the proposed REA is an attractive alternative solution which outperforms other modern algorithms and, at the same time, has a reduced complexity in comparison with them.

\section{CONCLUSIONS}

In this paper, the joint state and parameter robust estimation algorithm for stochastic linear time-varying systems, in presence of non-Gaussian noises has been proposed. The proposed algorithm has been used to solve the joint estimation problem of linear stochastic models where the conventional approaches fails. Since the system is described with a 
stochastic model with variable parameters, the natural frame for identification were robust Kalman filters. Because of their good features in robust filtering, the modified extended Masreliez-Martin filter was used as a basis in formulating the joint robust estimator of linear stochastic systems. The benefits of the proposed robust algorithms were illustrated through intensive simulations.

Acknowledgement: This research has been supported by the Serbian Ministry of Education, Science and Technological Development through projects TR33026 and TR33027.

\section{REFERENCES}

[1] V. Filipovic, N. Nedic, and V. Stojanovic, "Robust identification of pneumatic servo actuators in the real situations", Forschung im Ingenieurwesen - Engineering Research, vol. 75, no. 4, pp. 183-196, 2011.

[2] R.E. Kalman, "New Approach to Linear Filtering and Prediction Problems", Transactions of the ASME Journal of Basic Engineering, vol. 82, no 1, pp. 35-45, 1960.

[3] Y. H. Chang, Q. Hu, and C.J. Tomlin, "Secure estimation based Kalman Filter for cyber-physical systems against sensor attacks", Automatica, vol. 95, pp. 399-412, 2018

[4] L. Chang, F. Zha, and F. Qin, "Indirect Kalman Filtering Based Attitude Estimation for Low-Cost Attitude and Heading Reference Systems", IEEE/ASME Transactions on Mechatronics, vol. 22, no. 4, pp. $1850-1858,2017$.

[5] D. Viegas, P. Batista, P. Oliveira and Carlos Silvestre, "Discrete-time distributed Kalman filter design for formations of autonomous vehicles", Control Engineering Practice, vol. 75, pp. 55-68, 2018.

[6] K. Maes, A. Iliopoulos, W. Weijtjens, C. Devriendt, and G. Lombaert, "Dynamic strain estimation for fatigue assessment of an offshore monopile wind turbine using filtering and modal expansion algorithms", Mechanical Systems and Signal Processing, vols. 76-77, pp. 592-611, 2016.

[7] A. Cavallo, G. De Maria, C. Natale, S. Pirozzi, "Slipping detection and avoidance based on Kalman filter", Mechatronics, vol. 24, no. 5, pp. 489-499, 2014

[8] V. Stojanovic, and N. Nedic, "Identification of time-varying OE models in presence of non-Gaussian noise: Application to pneumatic servo drives", International Journal of Robust and Nonlinear Control, vol. 26, no. 18, pp. 3974-3995, 2016.

[9] M.T. Rodriguez, and S.P. Banks, "Linear, Time-varying Approximations to Nonlinear Dynamical Systems". Springer, Berlin, 2010.

[10] H. Cox, "On the estimation of state variables and parameters for noisy dynamic systems", IEEE Transactions on Automatic Control, vol. 9, no. 1, pp. 5-12, 1964.

[11] L. Ljung, "Asymptotic behavior of the extended Kalman filter as a parameter estimator for linear systems", IEEE Transactions on Automatic Control, vol. 24, no. 1, pp. 36-50, 1979.

[12] S. Aksoy, A. Muhurcu, and H. Kizmaz, "State and parameter estimation in induction motor using the Extended Kalman Filtering algorithm", in Proceedings of the International Symposium Modern Electric Power Systems, pp. 1-5, Wroclaw, Poland, September 2010

[13] G.L. Plett, "Extended Kalman filtering for battery management systems of LiPB-based HEV battery packs: Part 3. State and parameter estimation", Journal of Power Sources, vol. 134, no. 2, pp. 277-292, 2004.

[14] A. Carrassi, and S. Vannitsem, "State and parameter estimation with the extended Kalman filter: an alternative formulation of the model error dynamics", Quarterly Journal of the Royal Meteorological Society, vol. 137, no.655, pp. 435-451, 2011.

[15] J. Chen, R.J. Patton, Robust Model-Based Fault Diagnosis for Dynamic Systems. Springer, 1999.

[16] R. Isermann, "Fault-Diagnosis Applications: Model-Based Condition Monitoring: Actuators, Drives, Machinery, Plants, Sensors, and Fault-tolerant Systems", Springer, 2011.

[17] V. Barnet, and T. Lewis, Outliers in statistical data, 3rd ed. Wiley-Blackwell, New York, 1994.

[18] P.J. Huber, Robust statistics. Wiley, New York, 1981.

[19] C.J. Masreliez, and R.D. Martin, "Robust Bayesian estimation for the linear model and robustifying the Kalman filter", IEEE Transactions on Automatic Control, vol. 22, no.3, pp. 361-371, 1977.

[20] V. Stojanovic, and N. Nedic, "Robust Kalman filtering for nonlinear multivariable stochastic systems in the presence of non-Gaussian noise", International Journal of Robust and Nonlinear Control, vol. 26, no. 3, pp. 445-460, 2016. 